\title{
Deficiência de ferro na mulher adulta
}

\section{The iron deficiency in adult woman}

Lilian P. Rodrigues ${ }^{I}$

Silvia Regina P. F. Jorge ${ }^{2}$

\begin{abstract}
A anemia ferropriva é a doença nutricional de maior prevalência no mundo, atingindo $20 \%$ a 30\% da população. As mulheres, gestantes ou não, fazem parte dos grupos considerados vulneráveis. Entre as possiveis causas da anemia por deficiência de ferro, as perdas sanguineas menstruais constituem o principal fator de anemia ferropriva da mulher adulta. A proposta deste estudo é rever as causas da anemia na mulher adulta, principalmente decorrente de sangramentos uterinos, assim como o diagnóstico e adoção de medidas preventivas. Rev. Bras. Hematol. Hemoter. 2010; 32(Supl.2):49-52.
\end{abstract}

Palavras-chave: Anemia; deficiência de ferro; mulher adulta.

\section{Introdução}

A deficiência de ferro é considerada a desordem de origem nutricional de maior prevalência. Segundo a Organização Mundial de Saúde, 20\% a 30 \% da população mundial adulta apresenta deficiência de ferro, ${ }^{1}$ sendo as mulheres, gestantes ou não, juntamente com as crianças, consideradas grupos mais vulneráveis.

\section{Prevalência}

Em países industrializados, a prevalência de anemia é de $5 \%$ em mulheres não grávidas; já a deficiência de ferro varia entre $5 \%$ e $11 \% .^{2} \mathrm{Na}$ América Latina e Caribe, a anemia ferropriva atinge ao redor de $30 \%$ das mulheres não gestantes.

A prevalência da deficiência de ferro em mulheres entre 20 e 49 anos é de $11 \%$, de $5 \%$ entre 50 a 69 anos, e de $2 \%$ na faixa etária acima de 70 anos, ${ }^{2}$ sendo, portanto, mais evidente no período da menacme, associada à menorragia.

\section{Etiologia}

As causas da deficiência de ferro são basicamente a baixa ingesta, absorção deficiente e perdas sanguíneas, incluindo fluxo menstrual excessivo.

A anemia se desenvolve lentamente, depois de esgotadas as reservas de ferro no corpo e na medula óssea. Inicialmente ocorre depleção dos depósitos de ferro no organismo, o que se constata com a diminuição da ferritina. Em decorrência da redução das reservas, ocorre comprometimento da eritropoese, observado por valores inferiores da hemoglobina corpuscular média (HCM) e volume corpuscular médio (VCM). Sequencialmente observa-se a diminuição dos valores de hemoglobina e hematócrito, o que compromete a oxigenação tecidual, instalando-se então o quadro de anemia.

De maneira geral, as mulheres tendem a apresentar menores reservas de ferro que os homens devido ao fluxo menstrual, portanto, maior risco para anemia. As causas da anemia na mulher diferem de acordo com as suas diferentes fases da vida: infância, menacme, gravidez e climatério.

${ }^{1}$ Ginecologista e obstetra. Professora Assistente da Faculdade de Ciências Médicas da Santa Casa de São Paulo - São Paulo-SP.

${ }^{2}$ Ginecologista e obstetra. Professora Instrutora da Faculdade de Ciências Médicas da Santa Casa de São Paulo- São Paulo-SP.

Faculdade de Ciências Médicas da Santa Casa de São Paulo. Setor de Gestação de Alto Risco do Departamento de Obstetrícia e Ginecologia da Irmandade da Santa Casa de Misericórdia de São Paulo - São Paulo-SP.

Correspondência: Lilian de Paiva Rodrigues

Rua Dr. Cesário Mota Jr, 112 - Vila Buarque

01221-020 - São Paulo-SP - Brasil

Tel.: (55 11) 3222-4254; Fax.: (55 11) 2176-7384

E-mail:cvk@uol.com.br

Doi: 10.1590/S1516-84842010005000059 
Tabela 1. Causas da deficiência de ferro na mulher adulta

1. Dieta deficiente em ferro

2. Diminuição da absorção de ferro

3. Aumento das necessidades: gravidez, lactação

4. Perda sanguínea:

Genital

Menstruação excessiva

Miomas uterino

Adenomiose

Pólipo endometrial

Hiperplasia endometrial

Hemorragia uterina disfuncional

Complicações gravídicas (abortamento, gestação ectópica, neoplasia trofoblástica gestacional)

Tumores neoplásicos

Gastrointestinais

Parasitismo

Doação de sangue

Medicamentos (aspirina, anti-inflamatórios não hormonais)

As causas de deficiência de ferro mais frequentemente encontradas na mulher estão expostas na Tabela 1.

Na mulher adulta, durante o menacme, a quantidade de ferro perdido no período menstrual deve ser considerada.

Para que ocorra adequada eritropoese, a quantidade de ferro necessária é de $5 \mathrm{mg}$ a $10 \mathrm{mg} / \mathrm{dia}$. Se o fornecimento for inferior a 70\% deste valor, ocorre a depleção dos estoques, e, em aproximadamente quatro meses, a eritropoese passa a ser deficitária, o que acarreta queda de $1.0 \mathrm{~g} / \mathrm{dL}$ nos valores de hemoglobina. ${ }^{2}$

Estudos têm mostrado valores médios de perdas sanguíneas entre $25 \mathrm{~mL}$ e $30 \mathrm{~mL} /$ mês para o fluxo menstrual, o que representa perda mensal entre $12,5 \mathrm{mg}-15 \mathrm{mg}$ de ferro ou ainda, 0,4 mg-0,5 mg/dia, nos 28 dias. Quando se agregam as perdas basais, a perda total de ferro devido à menstruação é cerca de $1,25 \mathrm{mg} /$ dia. Isso significa que a necessidade de ferro em aproximadamente $50 \%$ de todas as mulheres é superior a esse valor. ${ }^{2-4}$

Os distúrbios menstruais constituem importante problema clínico, afetando cerca de $25 \%$ a $30 \%$ das mulheres durante sua vida reprodutiva. As perdas sanguíneas acima de $80 \mathrm{~mL}$ por fluxo menstrual são consideradas excessivas (menorragia)., ${ }^{3,4}$

A hemorragia uterina disfuncional, causa importante de menorragia, constitui-se em uma das queixas mais comuns nas mulheres desde o período da adolescência até a perimenopausa. Estima-se que 50\% das pacientes com sangramento uterino disfuncional tenham idade ao redor dos 45 anos, e que aproximadamente $20 \%$ sejam adolescentes. O sangramento uterino disfuncional usualmente está associado com função ovariana anormal e anovulação, manifestandose mais frequentemente por sangramento uterino abundante, irregular e indolor. Também pode ter origem endometrial, atribuída às alterações nos mecanismos neuroendócrinos que controlam a menstruação. ${ }^{3,4}$

\section{Repercussões clínicas}

A menorragia constitui-se em problema clínico e social relevante, visto que aproximadamente $20 \%$ das mulheres com queixas quanto a perdas menstruais referem interferência na qualidade de vida, com danos às atividades sociais e profissionais.

Associada a elevada frequência na mulher adulta, a deficiência de ferro é a principal causa de anemia nesta população, cujas repercussões clínicas já estão bem estabelecidas, decorrentes da hipóxia tecidual por transporte de oxigênio deficitário.

\section{Diagnóstico}

Em mulheres em idade fértil, a principal causa de deficiência de ferro é o hiperfluxo menstrual. ${ }^{5}$

No que se refere à avaliação das perdas menstruais, a anamnese deve ser dirigida no sentido de se caracterizarem os ciclos menstruais em relação à duração do fluxo, regularidade, o volume excessivo e presença de coágulos. Uma forma prática é o questionamento quanto ao número de absorventes utilizados diariamente e o intervalo de troca. ${ }^{6,7}$

Devem ser consideradas outras causas ginecológicas que podem contribuir para as perdas vaginais irregulares e abundantes, como pólipos, miomas uterinos, tumores pélvicos. Também igualmente importante é a investigação de sangramento intermenstrual e pós-coital..$^{6-8}$

O uso de medicamentos como hormônios exógenos, ácido acetilsalicílico, anticoagulantes, anticonvulsivantes, antidepressivos tricíclicos e digitálicos também deve ser investigado pela possibilidade de associação com quadros de menorragia. ${ }^{6-8}$

Outros fatores relacionados que devem ser avaliados é a concomitância com outros estados mórbidos, como: epilepsia, galactorreia, hirsutismo, tireopatia, doenças crônicas (diabete melito, doenças hepáticas e renais), discrasias sanguíneas, estado nutricional e hábitos de vida, como hábitos alimentares, excesso de atividade física, etilismo, tabagismo entre outros. ${ }^{8,9}$

O exame físico geral deve avaliar a presença das condições anteriormente citadas. Além do estado geral, medidas de peso e estatura que auxiliam na determinação de eventos puberais e distúrbios nutricionais devem ser avaliados. A presença de petéquias e equimoses pode revelar coagulopatias associadas. Endocrinopatias com presença de hirsutismo, virilização e bócio podem revelar disfunção tiroidiana, hiperplasia de supra-renal, Cushing, diabete mellitus e ovários policísticos entre outros. ${ }^{8,9}$

O exame pélvico deve avaliar a integridade do períneo, vagina e colo uterino quanto à presença de lesões ou traumas. 
A colheita da citologia oncológica cérvico-vaginal deve ser incluída, contudo a suspeita diagnóstica de câncer cervical pode ser estabelecida pela visualização da massa tumoral. $\mathrm{O}$ toque bimanual permite avaliação do tamanho e consistência uterina, assim como a presença de tumores pélvicos ou, ainda, de eventual gravidez..$^{6-9}$

Quando a intensidade da anemia for desproporcional à perda menstrual, outra causa deve ser investigada. ${ }^{5} \mathrm{Em}$ aproximadamente $20 \%$ das mulheres ocorre sangramento do trato gastrointestinal conjuntamente. A investigação deve ser considerada na presença de dor abdominal, dispepsia, refluxo, perda de peso, história familiar de câncer digestivo, presença de anemia refratária ou recorrente; perda sanguínea menstrual referida desproporcional à intensidade da anemia e história prévia de doença péptica (infecção pelo H. pylori, doença celíaca). ${ }^{5}$

\section{Exames complementares}

No roteiro diagnóstico deve-se afastar inicialmente a possibilidade de gestação. A avaliação laboratorial para a pesquisa de deficiência de ferro encontra-se em capítulo específico sobre diagnóstico.

Quanto a avaliação laboratorial da menorragia, deve-se incluir, além dos índices hematológicos, quantificação hormonal, incluindo gonadotrofinas hipofisárias, esteroides sexuais e função tiereoidiana. A colheita da citologia oncológica deve ser incluída, mas a suspeita de câncer cervical pode ser feita pela visualização do tumor. ${ }^{8,9}$

A ultrassonografia transvaginal fornece informações sobre o volume uterino e ovariano, eco endometrial bem como a presença de nódulos, cistos ou tumorações pélvicas. A investigação endometrial (histeroscopia diagnóstica, biópsia de endométrio) está indicada diante de alterações do eco endometrial, nos sangramentos intermenstrual e da pósmenopausa, e nos casos de falha terapêutica. ${ }^{6}$

Na Tabela 2 encontram-se expostos os exames complementares auxiliares para o diagnóstico das menorragias.

Tabela 2. Exames complementares para a pesquisa dos sangramentos genitais.

\section{Hemograma}

Ferritina

B hcG

Citologia cervical

FSH, LH, Progesterona, Estradiol, Prolactina

TSH, T4 livre

Testosterona total

Sulfato de Deidroepiandrosterona (DHEA-S)

Coagulograma

Ultrassonografia transvaginal

Histeroscopia diagnóstica

Biópsia endometrial

\section{Prevenção}

Como já exposto, nas mulheres em idade fértil, as perdas sanguíneas menstruais representam necessidades diárias de ferro entre $1,4 \mathrm{mg}$ a $2 \mathrm{mg}$ /dia, sendo que, para $10 \%$, com sangramentos maiores, as necessidades diárias podem chegar a $3 \mathrm{mg}$ ou mais. ${ }^{4,5,10}$

Assim, como medidas preventivas, a Organização Panamericana de Saúde (OPS) e a Organização Mundial de Saúde (OMS) sugerem algumas ações, como orientação nutricional, com ingesta adequada de ferro, principalmente de origem animal (ferro heme), além de fortificação com ferro de alimentos de baixo custo, consumidos pela população. ${ }^{11,12}$

Especial atenção deve ser dada às perdas sanguíneas menstruais. Há necessidade de vigilância permanente e suplementação medicamentosa de ferro nas mulheres com sangramento uterino anormal. A duração e intervalo variam individualmente, de acordo com cada caso. ${ }^{10}$

A primeira opção de suplementação de ferro é pela via oral. Contudo, a adesão é baixa, reduzindo a eficácia de tal medida, principalmente em decorrência dos efeitos colaterais indesejados causados pela ingestão do ferro por via oral.

Assim, a suplementação intermitente, semanal, tem sido proposta, na última década, para grupos vúneráveis, como as mulheres na menacme, demostrando-se mais efetiva como medida alternativa para prevenção da deficiência de ferro, apesar de não existirem ainda recomendações padronizadas definindo a melhor dose e frequência. ${ }^{13-15}$

A identificação da causa de deficiência de ferro e instituição de medidas corretivas, quando possíveis, são ações essenciais para a prevenção da instalação da anemia na mulher adulta.

Nos casos de sangramento uterino anormal, tratamento medicamentoso, com uso de compostos hormonais (contraceptivos orais combinados, progestogênios, análogos de GnRh, DIU liberador de progestogênio) e não hormonais (ácido tranexâmico, ácido mefenâmico) pode ser indicado, no intuito de redução das perdas ou suspensão do ciclo menstrual. ${ }^{6,8,9}$

Intervenções cirúrgicas, como curetagem uterina, ablação endometrial, miomectomias e histerectomías têm indicações precisas e individuais..$^{6,8,9}$

\section{Abstract}

Iron-deficiency anemia is the most common nutritional disease in the world, affecting from 20 to $30 \%$ of the population. Women, both pregnant and otherwise, are included as groups considered vulnerable. Among the possible causes of iron-deficiency anemia, menstruation blood loss is the main cause of iron-deficiency anemia in adult women. The purpose of this study was to review the causes of anemia in adult women, in particular in relation to uterine blood 
loss including the diagnosis and the adoption of preventive measures. Rev. Bras. Hematol. Hemoter. 2010;32(Supl.2):49-52.

Key words: Anemia; iron deficiency; adult woman.

\section{Referências Bibliográficas}

1. World Health Organization. Iron deficiency anaemia: assessment, prevention and control. A guide for programme managers. Geneva: World Health Organization; 2001. (WHO/NHD/01.3)

2. Dallman PR, Yip R, Johnson C. Prevalence and causes of anemia in the United States, 1976 to 1980. Am J Clin Nutr. 1984;39(3): 437-45.

3. Anemia Working Group Latin America. Compendio de guías latinoamericanas para el manejo de la anemia ferropénica. $2^{\mathrm{a}}$ ed. 2007.

4. Ballard L, Lyon DS, Jones JL. Inpatients with menometrorrhagia: etiologies, treatments and outcomes. South Med J. 2000;93(6): 571-4.

5. Tefferi A. Anemia in adults: a contemporary in adults approach to diagnosis. Mayo Clin Proc. 2003;78(10):1274-80.

6. Ginecologia endócrina: Manual de orientação. Ricardo Mello Marinho, Mauri José Piazza, João Pedro Junqueira Caetano ed. - São Paulo: Editora Ponto, 2003. v.01.168 p.

7. American College of Obstetricians and Gynecologists. ACOG Practice Bulletin $\mathrm{N}^{\circ}$ 14: anemia in pregnancy. Obstet Gynecol. 2008;112(1):201-7.

8. National Health Service Center for Reviews and Dissemination. Management of menorrhagia. Halifax, UK: University of York (Stott Brothers Ltd); 1995. Effective Health Care Bulletin, vol. $1(9)$.

9. Prentice A. Medical management of menorrhagia. BMJ. 1999; 319:1343-5.

10. Baker WF Jr. Iron deficiency in pregnancy, obstetrics and gynecology. Hematol Oncol Clin North Am. 2000;14(5):1061-77

11. Unicef/UNU/WHO/MI. Technical Workshop. Preventing iron deficiency in woman and children: background and consensus on key technical issues. New York: INF/MI; 1998.

12. World Health Organization. Iron deficiency anaemia. Assessement, prevention, and control. A guide for programme managers. WHO/ Unicef/UNU; 2001.

13. Gross R, Schultink W, Juliawati. Treatment of anaemia with weekly iron supplemention. Lancet. 1994;344(8925):821

14. Hallberg L. Combating iron deficiency: daily administration of iron is far superior to weekly administration. Am J Clin Nutr. 1998;68(2):213-7

15. Allen LH. Iron supplements: scientific issues concerning efficacy and implications for research and programs. J Nutr. 2002;132(4 Suppl):813S-9S

O tema foi sugerido e avaliado pelo coeditor deste fascículo educativo, Rodolfo Cançado, e pelo board interno da RBHH, e publicado após a concordância do editor, Milton Artur Ruiz.

Conflito de interesse: sem conflito de interesse

Recebido: 21/12/2009

Aceito: 16/01/2010 